\title{
The effect of the titanium nitride coating on bacterial adhesion on orthodontic stainless steel wires: in vivo study
}

\author{
Fariborz Amini', Abbas Bahador ${ }^{2}$, Bita Kiaee ${ }^{3}$ and Gita Kiaee ${ }^{4}$ \\ ${ }^{1}$ Professor of Department of Orthodontics, Islamic Azad University of Medical Science, Dental Branch, \\ Tehran, Iran \\ ${ }^{2}$ Professor of Department of Microbiology, Tehran University of Medical Sciences, Tehran, Iran \\ ${ }^{3}$ Orthodontic Resident, Department of Orthodontics, Islamic Azad University of Medical Science, Dental \\ Branch, Tehran, Iran \\ ${ }^{4}$ Department of Biomedical Engineering, Tufts University, MA, 02155, United States of America
}

\begin{abstract}
The aim of the current study was an in vivo evaluation of the bacterial adhesion on TiN coated orthodontic stainless steel wires in 20 patients (age15-25) undergoing fixed orthodontic treatment who have the indication for placement of $0.019 \times 0.025$ inch Stainless steel arch wires in both jaws. TiN coatings were formed on orthodontic stainless steel wires by physical vapor deposition (PVD), Then the accuracy of the procedure was detected using SEM microscopy (PHILIPS XL 30) at $\times 2000$ magnify. Each patient will serve as their own control, the coated arch wires will randomly be assigned to one jaw and the opposing jaw will receive a non-coated arch wires. After 4 weeks, the arch wires will be removed and cut at the same lengths $(20 \mathrm{~mm})$. The samples were placed in sterile dishes containing phosphate buffered saline. After separation of bacteria in trypsin (25\%) and ethylene di-amine teta-acetic solutions for 45 minutes, the diluted solution was cultured in blood agar and bacterial colony forming units were counted. According to the results, significant difference detected on colony count on TiN coated orthodontic stainless steel wire $\left(4 \pm 3.4 \times 10^{4}\right)$ compared to non- coated orthodontic stainless steel wire $\left(8 \pm 7.4 \times 10^{4}\right)(\mathrm{P}=0.03)$. These results suggested that the TiN coated orthodontic stainless steel wire decreased bacterial growth and enhanced oral hygiene.
\end{abstract}

KEY WORDS: BACTERIAL ADHESION, TITANIUM NITRIDE, STAINLESS STEEL, ORTHODONTIC WIRE

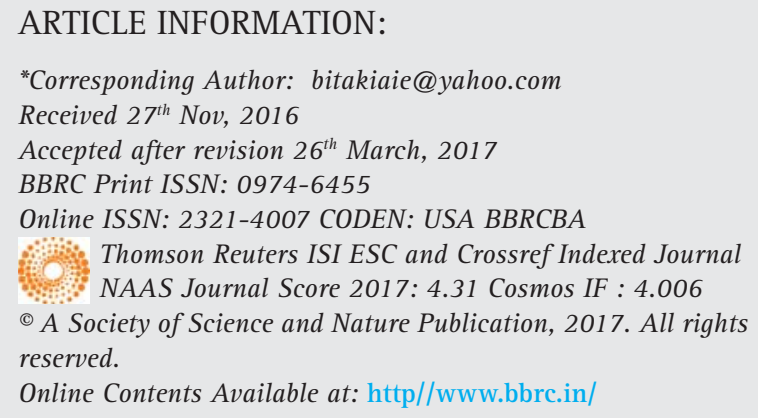




\section{INTRODUCTION}

Nowadays more people want orthodontic treatment to enhance their quality of life and obtain beautiful and healthy smile but placement of orthodontic appliances like brackets, tubes, band material, ligating materials and arch-wires inhibit the maintenance of a proper oral hygiene and are liable for microbial adhesion and create new retentive areas for plaque and debris, which in turn pre-disposes the patients to increased microbial burden and possibility of subsequent side effects such as gingival inflammation and white spot lesions (Papaioannou et al. 2007). Moreover, orthodontic appliances severely hamper the efficacy of tooth brushing, reduce the selfclearance by saliva, change the composition of the oral flora, increase the amount of oral biofilm formed and the colonization of oral surfaces by cariogenic and periodontopathogenic bacteria. These factors strongly complicate orthodontic treatment, and illustrate that the need for oral biofilm control is even greater during orthodontic treatment than usual (Pejda et al. 2013).

The incidence of enamel demineralization and periodontal disease after fixed orthodontic treatment can involve up to $50 \%$ of patients (Nascimento et al. 2014). Increased oral microbiota of Streptococcus mutans and Lactobacillus is associated with the onset of tooth demineralization and periodontal disease. Among different fixed orthodontic appliances, wires could play a significant role in enamel demineralization because they are present throughout the period of orthodontic treatment. Areas of contact between the wire and brackets provide a unique environment that impedes proper access to tooth surfaces for cleaning, (Mhaske et al. 2015).

Eliades et al. (1995) earlier had reported that stainless steel represented the highest critical surface tension and energy and can be expected to have higher plaque retaining capacity. In addition, stainless steel was found to induce specific changes in the oral environment such as decreased $\mathrm{pH}$, increased plaque accumulation, and elevated Streptococcus mutans and Lactobacillus acidophilus colonization (Sug-Joon et al. 2007). Photocatalytic may provide a novel tool for the prevention of bacterial contamination and disinfection (Chun et al. 2007). It is reported, the surface modification of orthodontic wires with photocatalytic can be used to prevent the development of dental plaque during orthodontic treatment (Chun et al. 2007). The orthodontic wires used in dental treatments supply a good habitat for oral microorganisms sufficient to cause dental caries or even periodontal diseases. More successful results can be achieved by reducing the chances for oral microorganisms to adhere to the surfaces of teeth and orthodontic wires. Therefore, the surface modification of orthodontic wires with photocatalytic may provide more effective results in orthodontic treatments (Chun et al. 2007). Semiconductor particles such as titanium oxide, zinc oxide, tungsten oxide, cadmium sulfide, zinc sulfide, strontium titanate oxide, and iron oxide are considered ideal photocatalysts for these reactions (Chun et al. 2007). For instance, surface modification of orthodontic wires with silver can be used to prevent the accumulation of dental plaque and the development of dental caries during orthodontic treatment. Among these, titanium has attracted considerable attention and has been reported to be the most useful substance in organic degradation processes on account of its chemically stable properties and absence of harmful effects on humans (Kim et al. 2010 and Mhaske et al. 2015).

Titanium nitride (TiN) or nitrogen ion implanted coated dental materials have been investigated since the mid-1980s (Al Jabbari et al. 2012) and considered in various applications in dentistry such as implants, abutments, orthodontic wires, endodontic files, periodontal/oral hygiene instruments, and casting alloys for fixed restorations (Al Jabbari et al. 2012) but few studies exists on orthodontic wires are modified with TiN and tested for its antimicrobial properties. So, the aim of the current study was to determine the effect of the TiN coating on bacterial adhesion on orthodontic stainless steel wires.

\section{MATERIAL AND METHODS}

This in vivo study was done in Orthodontic department of Islamic Azad University of Medical Sciences, Tehran, Iran during 2015. A total 20 patients undergoing orthodontic treatment were included in the study. The patients aged between 15-25 years old. In each group 10 boys and 10 girls were allocated. Before the study, all patients were informed about the study duration and protocol (e.g. not application antibiotic and antibacterial mouthwash during the study) and filled and signed the agreement form.

\section{STUDY PROTOCOL}

The orthodontic wires used in this study were $0.019 \times$ 0.025 inch Stainless steel (G\&H Wire Company, USA) washed using Veb Hoch vacuum Dresden and ultrasonic device. Then the orthodontic wire was allocated into argon vacuum chamber at $10^{-3} \mathrm{mBar}$ for 60 minute, Nitrogen gas $10^{-5} \mathrm{mBar}$ for 20 minute. Then TiN coated on the Stainless steel orthodontic wire using electrolytic treatment system. First coatation was done using 600 volt and decreased to 200 volt in 10 minutes, continued for 20 minute. This procedure was taken 30 minutes at $180^{\circ} \mathrm{C}$. The titanium applied before nitrogen gas expo- 
sure to enhance coated wires. Then the accuracy of the procedure was detected using SEM microscopy (PHILIPS XL 30) at $\times 2000$ magnify (fig.1). Then two types of the orthodontic wires (coated and non-coated) were used in each patient, the coated arch wire will randomly be assigned to one jaw and the opposing jaw will receive a non-coated arch wire. Then wires were removed after 4 weeks.

\section{COLONY COUNT}

Wires were removed and cut at the same lengths (20 $\mathrm{mm}$ ) and stored in sterile dishes containing phosphate buffered saline. Then samples mixed with trypsin (25\%) and ethylene di-amine teta-acetic solutions for $45 \mathrm{~min}$ utes to separate bacteria from orthodontic wires. Then suspension diluted with $2.99 \mathrm{~mL}$ saline to volume of 3 $\mathrm{mL}$. This procedure was done to achieve final dilution $(10 \mu \mathrm{L})$ and incubated on blood agar plate at $37^{\circ} \mathrm{C}$ for 24 hours. Then colonies were counted in $1 \times 10^{4}$ (Kantoriski et al. 2006).

\section{STATISTICAL ANALYSIS}

Data for bacteria load was analyzed by one way analysis of variance (ANOVA) using SPSS 16.0 for Windows and is presented as mean \pm Std. Deviation. For treatments showing a main effect by ANOVA, means were compared using Tukey HSD test. $\mathrm{P}<0.05$ was considered as significant differences between treatments.

\section{RESULTS AND DISCUSSION}

The result of the bacterial load on the orthodontic stainless steel wire is presented in table 1 . According to the results, significant difference detected on colony count on TiN coated orthodontic stainless steel wire $(4 \pm 3.4$ $\left.\times 10^{4}\right)$ compared to non-coated orthodontic stainless steel wire $\left(8 \pm 7.4 \times 10^{4}\right)(\mathrm{P}=0.03)$.

Orthodontic appliances frequently encroach on the gingival sulcus and act as an obstacle for maintaining the oral hygiene. Increase in inflammation is noted immediately after placement of fixed orthodontic appliances. The level of oral hygiene during treatment has a direct influence on periodontal status. Even with excellent oral hygiene, the majority of patients usually develop moder- ate gingivitis within few months after placement of the appliances. These changes are generally transient and are reversible with no permanent damage to the periodontal tissues (Shashidhar et al. 2015).

Patients with orthodontic appliances apart from providing new retention areas of bacterial colonization will also experience oral ecologic changes such as low salivary $\mathrm{pH}$, increased retention of food particles which may lead to increase dental decay (Van Gastel et al. 2009). As observed in this study, colony count on TiN coated stainless steel orthodontic wire $\left(4 \pm 3.4 \times 10^{4}\right)$ compared to non-coated orthodontic stainless steel wire (8 $\left.\pm 7.4 \times 10^{4}\right)(\mathrm{P}=0.03)$. These results suggested the coated stainless steel orthodontic wire with TiN decreased bacterial growth and enhanced oral hygiene.

Complicated appliance designs with loops and auxiliary arch wires create areas that are difficult to clean and may therefore enhance biofilm formation (Ogaard, 2008). The inter-bracket part of arch wires is relatively distant from the enamel surface and gingival tissues, and biofilms formed here may also be considered relatively harmless to the enamel and gingival tissues. Moreover, biofilms on these parts are easier to remove by brushing, compared with those formed on brackets, adhesives, and ligating devices. Biofilms on the arch wires ligated in the bracket slot may however, compromise the efficiency of the sliding mechanics (Eliades and Bourauel, 2005).

Studies assessed the antibacterial activity of TiN coatings and concluded that there is no difference between $\mathrm{Ti}$ and nitrified $\mathrm{Ti}$ for adherence of Streptococcus mutans (Tamura et al. 2002), Porphyromonas gingivalis, and Actinobacillus actinomycetemcomitans (Yoshinari et al. 2001). In contrast, the adherence of Streptococcus mutans and Streptococcus sanguis was significantly reduced on TiN surfaces compared to polished ones (Grössner-Schreiber et al. 2001). The same favorable results were found for titanium plasma sprayed compared to uncoated titanium plasma sprayed where Streptococcus pyogenes and Streptococcus sanguinis bacteria demonstrated decreased bacterial adhesion and proliferation (Annunziata et al. 2011). Two studies based on in vivo data confirmed the positive effect of TiN coating on the antibacterial activity of TiN surfaces. The results of Scarano et al. (2003) showed that implants coated with TiN illustrated a minor quantity of the surface covered by bacteria after a 24 -h exposure to the oral cavity while

Table 1 . the bacterial load on the stainless steel orthodontic wire $(\mathrm{N}=20$, results is $1 \times 104$ )

\begin{tabular}{|l|l|l|l|l|}
\hline Groups & Mean \pm Sd & Min & Max & P value \\
\cline { 1 - 4 } TiN coated orthodontic stainless steel wire & $4 \pm 3.4$ & 0.1 & 12.7 & \multirow{2}{*}{0.03} \\
\cline { 1 - 3 } Non -coated orthodontic stainless steel wire & $8 \pm 7.4$ & 0.3 & 34.5 & \\
\hline
\end{tabular}




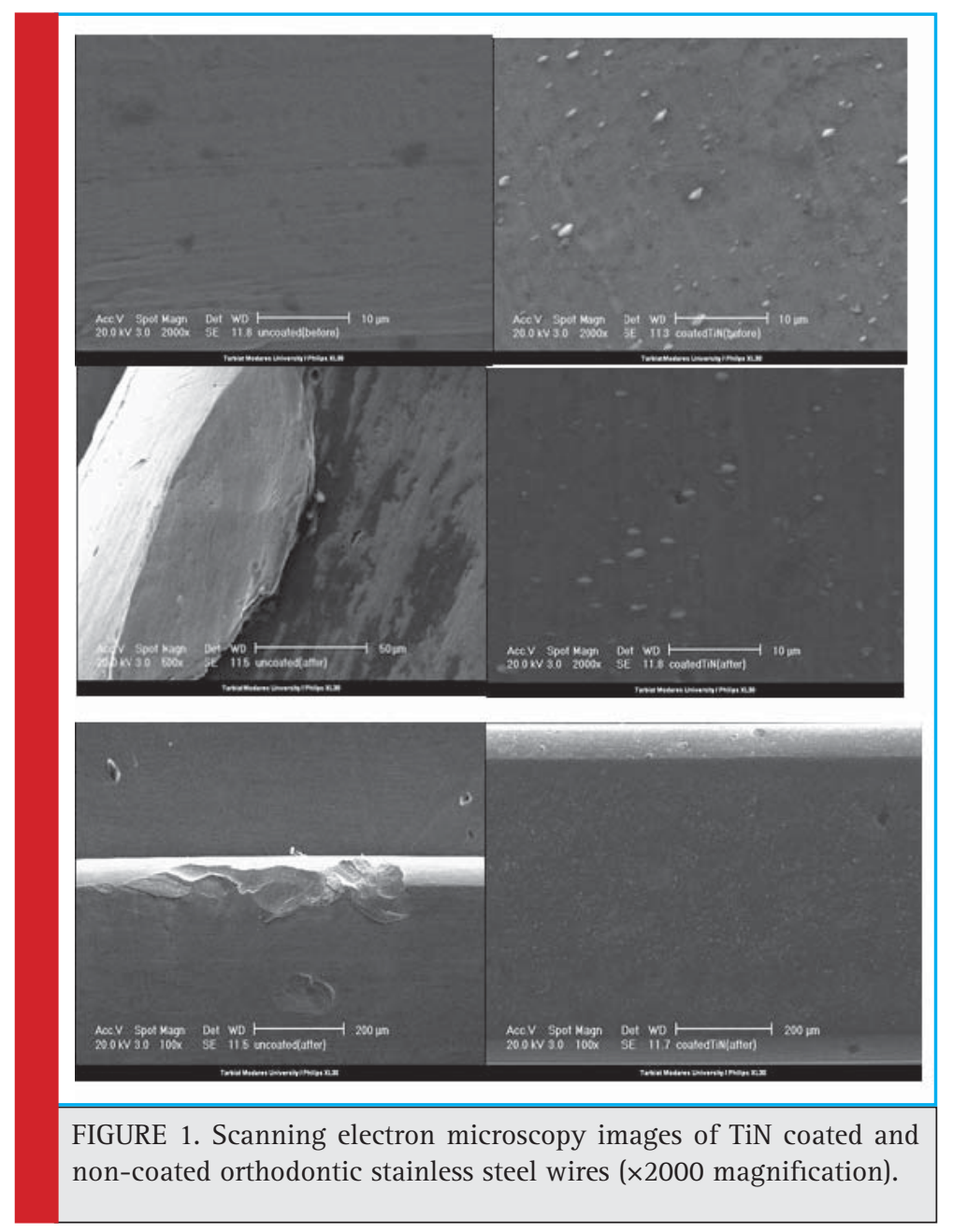

a smaller bacterial quantity were found on TiN glass sheets compared to pure titanium coated glass sheets after $60 \mathrm{~h}$ of intraoral exposure (Grössner-Schreiber et al. 2004). TiN coatings seem to have a beneficial effect on antibacterial activity inhibiting the formation of microbial plaque, minimizing the adverse effects of periimplantitis on implant longevity. The aforementioned antibacterial activity combined with the wear resistance to scaling treatment points out that the application of TiN coatings to implant abutments holds promise and is worthwhile for further scientific research and optimization.

Iijima et al. (2010) studied the corrosion properties of nitrogen ion implanted NiTi wires (Neo Sentalloy Ionguard) compared to non-ion implanted NiTi (Neo Sentalloy). The two wires were examined utilizing potentiodynamic polarization measurements, which were completed in both artificial saliva and a fluoride mouth rinse solution, as it has been hypothesized that the acidic environment created by fluoride may dissolve the TiN coating.
Ion implantation is another method used to modify the surface of materials. It consists of a low temperature process in which ions penetrate the surface of a material and modify it instead of coating it (Narayan, 2009). This technique has been used in orthodontics for different purposes.

It is reported, the sliding friction force between 0.014 inch NiTi wire and the bracket ranged from 2.94 to $31.42 \mathrm{~N}$ in a simulated intra-oral environment. After the mechanical test, the presence of deeper binding scratches was observed by SEM (Chang et al. 2013). Doshi and Bhad-Patil found a positive correlation between roughness and frictional resistance of orthodontic wires and brackets (Doshi and Bhad-Patil, 2011). It is reported, the surface roughness value was $19.2 \mathrm{~nm}$ for the untreated NiTi wire and $21.9 \mathrm{~nm}$ for the TiN/Ti-coated wire. The slight increase in roughness would not significantly increase the frictional resistance during sliding motions. Owing to high wear resistance, a TiN coating to improve the wear and fatigue resistance of hip prostheses (Piscanec et al. 2004). 
Other study reported that the TiN coating on the NiTi alloy reduced the friction coefficient and improves wear resistance by sliding wear tests. Bacterial adhesion on medical devices has a strong influence on the long-term outcomes of clinical applications. An antibacterial ability is important in dental applications. Sukontapatipark et al. (2001) evaluated the surface of fixed orthodontic appliances in the oral cavity. After a 3-week tooth bonding period, results from SEM observation indicate that bacterial colonization had occurred on orthodontic brackets and ligature surfaces. Orthodontic treatment procedures are associated with bacteremia, in which aerobic and anaerobic bacteria were isolated from blood samples of these patients. A separate study exposed (Lucas et al. 2002). TiN-coated surfaces showed a significant reduction in the presence of bacteria, in comparison with Ti substrate (Scarano et al. 2003).

\section{CONCLUSION}

In conclusion these results suggested that the coated orthodontic stainless steel wire with titanium nitride decreased bacterial growth and enhanced oral hygiene. Orthodontic surface treatment is an important area of active research. A myriad of materials and techniques have been implemented to modify the surfaces of dental materials. However, today only a few are being used in clinical orthodontics, especially in areas such as friction control and reduction of bacterial adhesion.

\section{REFERENCES}

Annunziata, M.; Oliva, A.; Basile, M.A.; Giordano, M.; Mazzola, N.; Rizzo, A.; Lanza, A.; Guida, L. (2011)The effects of titanium nitride-coating on the topographic and biological features of TPS implant surfaces. J. Dent. 39, 720-728.

Chang C.J., Lee T.M., Liu J.K., (2013) The effect of bracket bevel design and oral environmental factors on frictional resistance, Angle Orthodontist 83 956-965.

Cho M, Chung H, Choi W, Yoon J. (2004) Linear correlation between inactivation of $\mathrm{E}$. coli and $\mathrm{OH}$ radical concentration in TiO2 photocatalytic disinfection. Water Res. 38: 1069-1077.

Chun MJ, Shim E, Kho EH, Park KJ, Jung J, Jin KM, Kim B, Lee KH, Cho DL, Bai DH, Lee S, Hwang HS, Ohk SH. (2007) Surface modification of orthodontic wires with photocatalytic titanium oxide for its antiadherent and antibacterial properties. Angle Orthodontist 77(3): 483-488.

Doshi U.H., Bhad-Patil W.A. (2011) Static frictional force and surface roughness of various bracket and wire combinations, American Journal of Orthodontics and Dentofacial Orthopedics 139 74-79.

Groessner-Schreiber, B.; Hannig, M.; Dück, A.; Griepentrog, M.; Wenderoth, D.F. (2004) Do different implant surfaces exposed in the oral cavity of humans show different biofilm compositions and activities? Eur. J. Oral Sci. 112, 516-522.

Grössner-Schreiber, B.; Griepentrog, M.; Haustein, I. (2001) Müller, W.D.; Lange, K.P.; Briedigkeit, H.; Göbel, U.B. Plaque formation on surface modified dental implants: An in vitro study. Clin. Oral Implant. Res. 12, 543-551.

Iijima, M.; Yuasa, T.; Endo, K.; Muguruma, T.; Ohno, H.; Mizoguchi, I. (2010) Corrosion behavior of ion implanted nickeltitanium orthodontic wire in fluoride mouth rinse solutions. Dent. Mater. J. 29, 53-58.

Kantoriski KZ, Scotti R, Felipe L. (2006) Surface roughness and Bacterial Adherence.Cience Odontol bras. 9(4):12-17.

Lucas V.S., Omar J., Vieira A., Roberts G.J. (2002) The relationship between odontogenic bacteraemia and orthodontic treatment procedures, European Journal of Orthodontics 24 293-301.

Nakajima A, Koizumi S, Watanabe T, Hashimoto K. (2000) Photoinduced amphiphilic surface on polycrystalline anatase TiO2 thin films. Langmuir. 16:7048-7050.

Narayan, R. (2009) Biomedical Materials; Springer: New York, NY, USA.

Nascimento LEAG, Souza MMG, Azevedo ARP, Maia LC. (2014) Are self-ligating brackets related to less formation of Streptococcus mutans colonies? A systematic review. Dental Press J Orthod. 19(1):60-8.

Papaioannou W, Gizani S, Nassika M, Kontou E, Nakou M. (2007) Adhesion of Streptococcus mutans to different types of brackets. Angle Orthod ; 77(6):1090-5.

Pejda S, Varga ML, Milosevic SA, Mestrovic S, Slaj M, Repic d, Bosnjak A. (2013) Clinical and microbiological parameters in patients with self-ligating and conventional brackets during early phase of orthodontic treatment. Angle Orthod. 83(1):133-9.

Piscanec S., Ciacchi L.C., Vesselli E., Comelli G., Sbaizero O., Meriani S., Vita A.D. (2004) Bioactivity of TiN-coated titanium implants, Acta Materialia 52 1237-1245.

Scarano A., Piattelli M., Vrespa G., Caputi S., Piattelli A. (2003) Bacterial adhesion on titanium nitride-coated and uncoated implants: an in vivo human study, Journal of Oral Implantology 29 80-85.

Scarano, A.; Piattelli, M.; Vrespa, G.; Caputi, S.; Piattelli, A. (2003) Bacterial adhesion on titanium nitride-coated and uncoated implants: An in vivo human study. J. Oral Implant. 29, 80-85.

Shashidhar P, Sahitya M, Sunil T, Murthy AR, Rani MS. (2015) A comparative evaluation of adherence of microorganism to different types of brackets: a scanning electron microscopic study. Journal of International Oral Health 7(9):25-30

Sukontapatipark W., El-Agroudi M.A., Selliseth N.J., Thunold K., Selvig Eur K.A. (2001) Bacterial colonization associated with fixed orthodontic appliances: A scanning electron microscopy study, European Journal of Orthodontics 23 475-484.

Tamura, Y.; Yokoyama, A.; Watari, F.; Kawasaki, T. (2002) Surface properties and biocompatibility of nitrided titanium 
for abrasion resistant implant materials. Dent. Mater. J. 21, 355-372.

Van Gastel Paolantonio M, di Girolamo G, Pedrazzoli V. (2009) Microbial Adhesion on Different Bracket Types in vitro. Angle Orthod. 79: 915-921.

Yamagishi M, Kuriki S, Song PK, Shigesato Y. Thin film TiO2 photocatalyst deposited by reactive magnetron sputtering. Thin Solid Films. 2003;442:227-231.

Yamaguichi M, Terao Y, Ogawa T, Takahashi T, Hamadas, Kawabata S. Role of streptococcus sanguinis sortase A in bacterial colonization. Microbes Infect. 2006; 8:2791-6.

Yoshinari, M.; Oda, Y.; Kato, T.; Okuda, K. Influence of surface modifications to titanium on antibacterial activity in vitro. Biomaterials 2001, 22, 2043-2048.

Mhaske AR, Shetty PC, Bhat NS, Ramachandra CS, Laxmikanth SM, Nagarahalli K, Tekale PD. Antiadherent and antibacterial properties of stainless steel and NiTi orthodontic wires coated with silver against Lactobacillus acidophilus-an in vitro study. Progress in Orthodontics (2015) 16:40. DOI 10.1186/s40510015-0110-0
Eliades T, Eliades G, Brantley WA. Microbial attachment on orthodontic appliances. I. Wettability and early pellicle formation on bracket materials. Am J Orthod Dentofacial Orthop. 1995;108:351-60.

Kim G, Rho Y, Park S, Kim H, Son S, Kim H et al. The biocompatibility of self-assembled brush polymers bearing glycine derivatives. Biomaterials 2010;31:3816-3826.

Sug-Joon A, Bum-Soon L, Shin-Jae L. Prevalence of cariogenic streptococci on incisor brackets detected by polymerase chain reaction. Am J Orthod Dentofacial Orthop. 2007;131:736-41.

Al Jabbari YS, Fehrman J, Barnes AC, Zapf AM, Zinelis S, Berzins DW. Titanium nitride and nitrogen ion implanted coated dental materials. Coatings 2012, 2, 160-178; doi:10.3390/coatings2030160

Ogaard B. White spot lesions during orthodontic treatment: mechanisms and fluoride preventive aspects Seminars in Orthodontics 2008;14:183-193.

Eliades T, Bourauel C. Intraoral aging of orthodontic materials: the picture we miss and its clinical relevance. Am J Orthod Dentofacial Orthop 2005;127:403-412. 